\title{
Educación y Derechos Humanos en mujeres adultas
}

\author{
Education and Human Rights in adult women
}

\section{Como citar el artículo}

Navas, R., (2019). Educación y Derechos Humanos en mujeres adultas. Un análisis confirmatorio con sistemas de ecuaciones estructurales. Revista Naturaleza, Sociedad y Ambiente, 6 (1), 89-96 DOI: https://doi.org/10.37533/cunsurori.v6i1.43

\section{Randolfo Alfaro Navas}

Licenciado en Pedagogía en administración Educativa, Maestría en Docencia Universitaria con énfasis en Andragogia

Recibido: 9 de agosto de 2019 / Aceptado: 6 de noviembre de 2019

Disponible en internet el 29 de noviembre de 2019

*Autor para correspondencia. Correo electrónico: idadifigueroa@gmail.com

\begin{abstract}
Resumen
La investigación se realizó en el Municipio de Yupiltepeque del Departamento de Jutiapa, con la finalidad de analizar el papel que ejercen los derechos humanos en ese Municipio y como son orientadas las mujeres adultas de los 20 a los 40 años el método que se utilizo fue el estudio de caso, y el cuestionario fue el instrumento para la recopilación de datos válidos y confiables, que permitieran dar respuesta a los objetivos planteados, se realizó el análisis de distintos autores para identificar el papel de los derechos humanos en las arias marginales de nuestro municipio y determinar cómo ese tipo de población desconoce totalmente los derechos que le asisten según los acuerdos internaciones y de nuestra constitución.
\end{abstract}

Palabras clave: mujeres, derechos, municipio, humanos y años

\section{Abstract}

The investigation was conducted in the Municipality of Yupiltepeque of the Department of Jutiapa, with the purpose of analyzing the role of human rights in that municipality and how adult women are oriented from 20 to 40 years old. The method used was the case study, and the questionnaire was the instrument for the collection of valid and reliable data, which allowed to respond to the objectives set, the analysis of different authors was carried out to identify the role of human rights in the marginal arias of our municipality and determine how this type of population is totally unaware of the rights that assist it according to international agreements and our constitution.

Keywords: women, rights, municipaly, humans, years 


\section{Introducción}

El trabajo de investigación tiene como propósito determinar los aspectos relacionados entre educación y derechos humanos en mujeres adultas en el Municipio de Yupiltepeque, Jutiapa; El planteamiento del problema se define como en el Municipio de Yupiltepeque del Departamento de Jutiapa, las personas adultas deben de tener una educación formal sistematizada al menos con el nivel básico para poder entender y comprender de forma adecuada los beneficios, y responsabilidades que conllevan los derechos humanos. Los Objetivos de la Investigación son determinar la carencia de educación formal y no formal sobre materia de derechos humanos que tienen las mujeres adultas en el municipio de Yupiltepeque Jutiapa los deseos de querer conocer qué porcentaje de la población desconoce este término.

\section{Fundamento Teórico}

La oficina de los derechos humanos debe capacitar a las mujeres adultas sobre los beneficios y las responsabilidades que los ciudadanos de nuestro país tienen en relación con sus necesidades, ya que las personas en las edades adultas no tuvieron esa oportunidad $y$ es interesante que sean capacitados para evitar que por el desconocimiento sean violados sus derechos. Según (Mauro, 1995): mejoran los índices de violaciones en derechos humanos con las capacitaciones que puedan recibir.

La educación formal, es el proceso integral correlacionado que abarca desde la educación primaria hasta la educación secundaria y la educación superior, y que conlleva una intención deliberada y sistemática que se concretiza en un currículo oficial, aplicado con definidos calendario y horario se trata pues de un tipo de educación regulada por los diferentes reglamentos internos dentro del proyecto educativo de cada colegio, intencional es formal porque tiene una planificación muy bien definida, porque tienen como intención principal la de educar y dar conocimientos a los alumnos, y planificado porque antes de comenzar cada curso, el colegio regula y planifica toda la acción educativa que va a ser transmitida en el mismo de esa cuenta que conocemos todo el conocimiento sistematizado (Rodríguez, 2006:6).

Educación formal básica. Es aquel ámbito de la educación que tiene carácter intencional, planificado y regulado, que abarca desde los primeros años de educación infantil hasta el final de la educación secundaria y conforma la escolarización obligatoria. En los grados de primero, segundo y tercero, tiene varias modalidades en el pensum de establecimientos públicos, privados en jornadas matutina, vespertina y algunas veces en jornadas nocturnas, así también educación acelerada para personas ya adultas, un proceso que ha venido a combatir el analfabetismo en nuestras comunidades, se cuenta con ciertas ventajas, como llevar un calendario de estudios, se planifica por temas, se calendariza para un mejor control en las actividades educativas (Salinas, 1995:11).

La educación formal incluye aquellos procesos de enseñanza-aprendizaje llevados a cabo en centros de educación o formación, con carácter estructurado según un programa con objetivos, metodología, técnicas, procedimientos, bibliografía, etc. y a cuyo término se obtiene un título o una certificación. El sistema educativo de un país se incluye dentro de esta categoría., como características básicas podríamos señalar que este tipo de educación se produce generalmente en espacio 
concreto y tiempo completo y que además con ella se transmite un título o diploma. Un ejemplo de este tipo de educación sería la recibida en las escuelas y en ella también se capacita en los primeros niveles orientación sobre derechos humanos, para que los niños vallan familiarizándose con estos procesos en materia de derechos humanos (Reyes, 2000:10).

Definición de Derechos humanos. Los derechos humanos son atributos universales, prioritarios e inherentes a toda persona, sin distinción de edad, razón por la cual no deben descrinarse las mujeres adultas de las edades de 20 a 40 años, 0 clase social (Torres y Bobadilla, 2004). Los derechos Humanos nacen con la humanidad misma, siempre se han encontrado presentes en la historia del ser humano, los cuales han ido evolucionando de acuerdo con cada época, en una lucha por poder hacer que se hagan y cumplan las leyes del respeto, la dignidad, el trabajo, y más (carbonel, 2002).

Faúdez (1996:16), afirma que los derechos humanos pueden definirse como las prorrogativas que, conforme el Derecho Internacional, tiene todo individuo frente a los órganos del poder para preservar su dignidad como ser humano, y cuya función es excluir la interferencia del estado en arias específicas de la vida individual, o asegurar la prestación de determinados servicios por parte del estado, para satisfacer para satisfacer sus necesidades básicas, y que reflejan las exigencias fundamentales que cada ser humano puede formular a la sociedad de que forma parte.

Baéz, (2007), afirma que los derechos humanos son facultades, prerrogativas, intereses y bienes de carácter civil, político, económico, social, cultural, psíquico e íntimo, que posee el ser humano, y que se reconocen en instrumentos jurídicos nacionales e internacionales. La importancia de los derechos humanos radica en que su finalidad es proteger la vida, la libertad, justicia, igualdad, la integridad de cada frente a la autoridad. En virtud que su única base es la condición propi8a de las personas, los derechos humanos son:

a) Universales, porque pertenecen a la humanidad en su totalidad

b) Inherentes a cada persona

c) Históricos, porque son resultado de la progresiva toma de conciencia de los seres humanos respecto de sus derechos y conquistas frente al abuso de poder público y privado.

El respaldo de la mujer en educación y derechos humanos. Según (García E. 1996), desde 1945 los diversos instrumentos legales de derechos humanos han dado su aval, a la educación en derechos humanos desde que la carta de las naciones unidas exigió la cooperación entre las naciones para el fomento y el estímulo, de esta cuenta la educación en materia de derechos humanos a la mujer esta establecida en los acuerdos mundiales de la declaración de los derechos humanos, por lo tanto deben de respetarse y sobre todo orientar a las mujeres sobre su fundamento legal y las obligaciones ni responsabilidades que tienen con la sociedad.

El valor de los derechos humanos. Según lo manifiesta (Roossevelt 1962), la declaración universal de los derechos humanos, dada el 10 de diciembre de 1948, fue una de las mejores ideas que pudo tener el ser humano ya que protege la vida y los derechos del ser humano, en virtud que es un derecho inherente de las personas de esa cuenta manifiesta que la declaración de los derechos humanos es como la carta magna de la humanidad, es por 
ello que es de suma importancia que en cualquier país del mundo se luche por el respeto hacia los derechos de las person Preguntas de investigación.

Según describe (García, 1999:19). El derecho del Niño la Niña y el Adolescente a vivir en familia se encuentra consagrado en diversas disposiciones del sistema internacional de derechos humanos. En especial, la vigencia y contenido de este derecho se desprende de los artículos (arts.) 5, 18, 19, 20 y 27 de la CDN; del artículo (art.) 19 del Pacto de San José de Costa Rica; del art. 15 del Protocolo de San Salvador y del art. 11 letra b) de las Reglas de Naciones Unidas.

Para la Protección de Menores Privados de Libertad. Estas normas reconocen a la familia como el medio prioritario en que debe desarrollarse el derecho al niño, la niña y el adolescente, afirman que el Estado debe proporcionar a que la familia cumpla la función de cuidado por ende, debe intervenir sólo cuando ella falle en este cometido; y establecen, por último, que la separación de su familia debe ser la última medida a adoptar y siempre que su interés superior lo autorice. Así, cabe destacar las Modalidades de Cuidado, sobre adolescentes. (guerrero, 2004:18).

Alcances de la actividad protectora de los derechos humanos. Mi postura sobre la procedencia de la actividad protectora de los derechos humanos, implica tener presente que la tutela de las prerrogativas humanas corresponde a toda autoridad del país. Incluso cometo el atrevimiento de afirmar que se trata de una obligación ciudadana que no admite la posibilidad de un monopolio a favor de una o varias autoridades o de crear un ámbito de impunidad conforme a los criterios judiciales que impiden la procedencia del amparo contra actos $u$ omisiones de particulares que violen prerrogativas humanas, sin soslayar la jurisprudencia que exenta al juez de distrito de violar derechos humanos (Espinoza, 1997:17).

El carácter universal de los derechos humanos. La universalidad según la que plantea menos problemas. Se dice que los derechos humanos son universales, No obstante, aun siendo el aspecto menos problemático, no deja de plantear alguna dificultad. Si los derechos son normas de un sistema, las consideraciones que se hagan respecto de los destinatarios de esas normas nos remitirán al examen del ámbito de aplicación de los sistemas en los que se insertan en ese sentido debe tener que ver, de alguna forma, con el ámbito de aplicación de los sistemas a los que esos derechos pertenecen. (Nino, 1989:08).

Los derechos humanos absolutos. La característica común a todos los derechos humanos es que son concebidos como límites infranqueables incluso para las mayorías. Constituyen un claro límite al poder de las mayorías políticas. Las mayorías políticas no pueden legítimamente privar de sus derechos a los individuos". Dicho límite no puede ser traspasado en ningún caso; de manera que los derechos "no pueden ser desplazados en ninguna circunstancia y por ninguna otra consideración moral" No obstante lo dicho, cree el autor que para dar solución a los conflictos entre derechos es posible suavizar este carácter absoluto (lo cual no deja de ser discutible, pues el término 'absoluto' no indica una propiedad gradual y, en consecuencia, es difícil que algo sea 'un poco absoluto Esto significa, en definitiva, que para resolver esos conflictos debemos tomar en consideración las consecuencias de que venza uno o el otro y decidirnos por uno de ellos (González, 2004:29). 
Derechos de primera generación. Los derechos civiles y políticos de primera generación, son todos aquellos que se refieren y que inciden sobre la expresión de la libertad de los individuos, fueron los primeros en ser reconocidos en el siglo XVIII en la independencia de los Estados Unidos y en la Revolución Francesa se trata de garantizar la libertad de las personas, proceden de la tradición constitucionalista liberal. Estos derechos están recogidos en la Declaración Universal de los Derechos Humanos de 1948, en donde se garantizan también la participación política de los ciudadanos y están inmersos el derecho al voto, a la seguridad, a la propiedad inclusive a la asociación de huelga, estos derechos deben de ser siempre respetados al ser humano, ya que son derechos inherentes de cada persona (Aguirre, 1987:27).

Derechos de segunda generación. Los derechos de segunda generación se incorporan a partir de una tradición de pensamiento humanista y socialista; son de naturaleza económica y social, e inciden sobre la expresión de igualdad de los individuos. Los derechos de primera generación defendían a los ciudadanos frente al poder del estado, pero ahora se exige cierta intervención del estado para garantizar un acceso igualitario a los derechos anteriormente citados, es decir, para compensar las desigualdades naturales creadas por las ventajas y desventajas de clases, etnia y religión que caracterizan las diferencias sociales de los individuos desde su propio nacimiento. Se pedía así que el estado que garantice el acceso a la educación, el trabajo, la salud, la protección social, creando las condiciones sociales que posibiliten un ejercicio real de las libertades en una sociedad donde no todos los hombres nacen iguales (Mirándola, 2009:30).
Derechos de tercera generación. Los llamados derechos de la solidaridad constituyen una tercera generación que se concretiza en la segunda mitad del siglo XX. Esta vez, su motor impulsor será la acción de determinados colectivos que reclaman legítimos derechos. Se comienzan a configurar en forma de declaraciones sectoriales que protegen los derechos de colectivos discriminados grupos de edad, minorías étnicas o religiosas, países del tercer mundo, que han salido afectados por alguna de las múltiples manifestaciones que cobra la discriminación económico social. En las dos últimas décadas del siglo que ya hemos abandonado, estos derechos han ido cobrando un papel cada vez más importante, y gracias a ellos se ha desarrollado concepto de diálogo Norte Sur, el respeto y la conservación de la diversidad cultural, la protección del medio ambiente, la conservación del patrimonio cultural del humanidad, su función principal promover relaciones pacíficas y Constructivas (García, 1999:31).

1. ¿Se aplicaran adecuadamente los derechos humanos?

2. ¿Cómo incide el desconocimiento de derechos humanos en las apersonas adultas?

3. ¿Qué instituciones velan por la aplicación de los derechos humanos?

4. ¿Cómo han sido capacitadas en materia de derechos humanos las personas en Yupiltepeque?

5. ¿Qué tipo de capacitaciones han recibido en materia de derechos humanos? 


\section{Metodología}

Estudio de casos, el estudio se realizara en el Barrio El Centro del Municipio de Yupiltepeque Jutiapa, para determinar si las mujeres adultas en las edades de 20 a 40 años han recibido alguna capacitación sobre los Derechos Humanos para poder acudir a las instancias en caso tener problemas con personas ajenas o con la familia o ya sea con su conviviente) se elaboró una encuesta con 5 preguntas y se entrevistaron en la calle, avenidas y sus domicilio a 50 personas de sexo femenino, para determinar si conocen en relación a los derechos que toda persona posee y que nunca pueden ser violentados.

\section{Resultados y discusión}

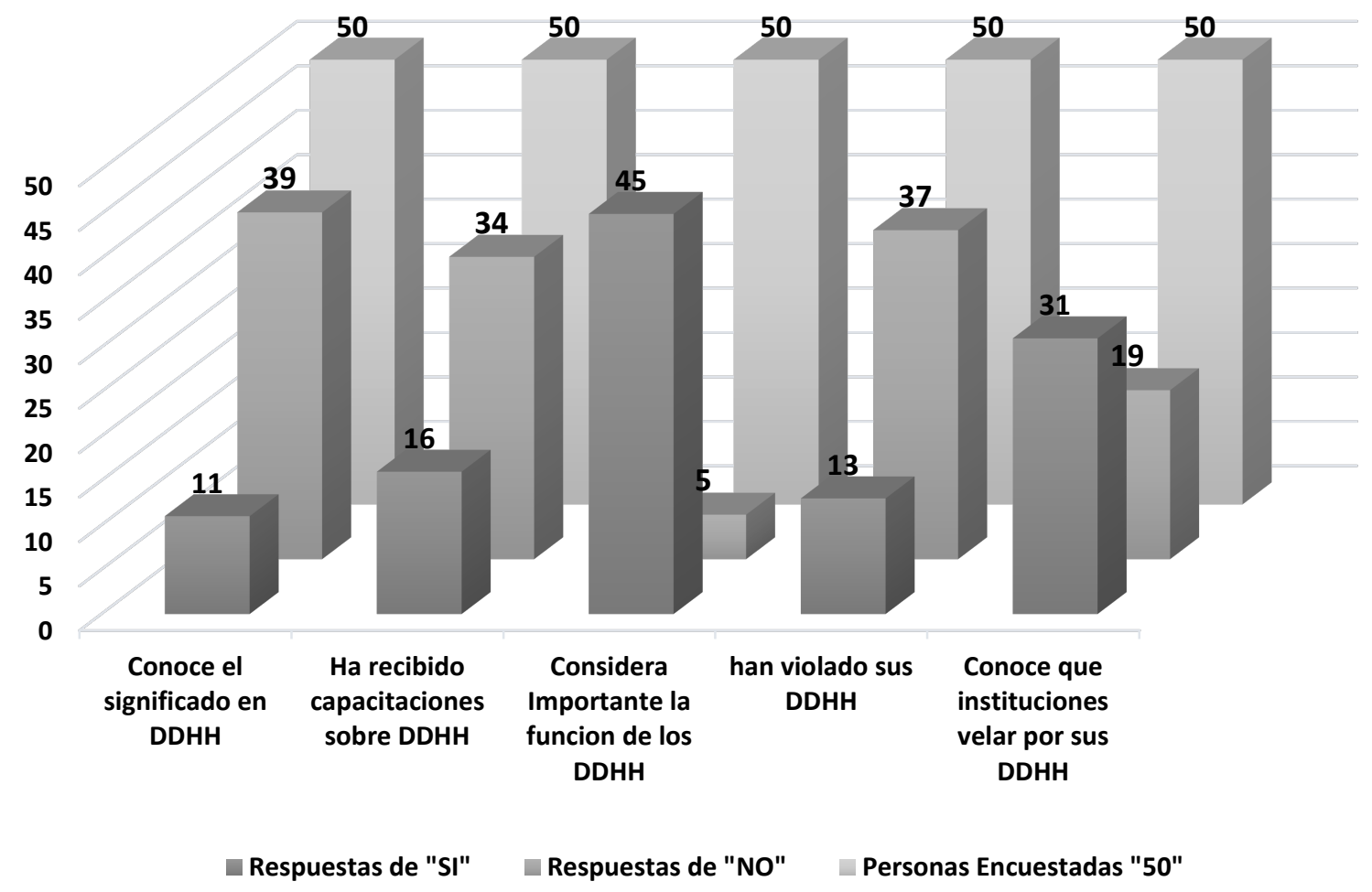

Cuadro No. 1

La encuesta se realizó a personas adultas de 20 a 40 años de sexo femenino en el Barrio El centro Yupiltepeque Jutiapa, con el propósito de establecer, si saben sobre el termino de derechos Humanos, si se les ha capacitado sobre el tema, si les parecen importantes, si en algún momento han sido objeto de viola- ciones y si conocen que instituciones velan en el país, para velar por el cumplimiento de los derechos humanos, se entrevistó a 50 personas que equivale al $100 \%$ del barrio el Centro del municipio de Yupiltepeque del departamento de Jutiapa. 


\section{Conclusiones}

Se determinó que las personas adultas en el Municipio de Yupiltepeque Jutiapa, la mayoría es equivalente al $78 \%$ comprenden el significado de los derechos humanos, pero que nunca han tenido ningún beneficio porque la institución no se preocupa por la población de escasos recursos y siempre benefician a otras personas

Se establece por medio de la encuesta aplicada a dichas personas que el $68 \%$ manifiesta que nunca han sido capacitadas en materia de los derechos humanos, en virtud que la mayoría no tuvieron la oportunidad de estudiar el nivel básico ni diversificado

De acuerdo a los resultados de la encuesta dirigida a los vecinos del Municipio de Yupiltepeque Jutiapa, el $90 \%$ manifiesta que si son importantes los derecho humanos en las personas, pues son derechos inherentes de los seres humanos pero que nunca se les da el debido cumplimiento por las instituciones encargadas de velar por ellos

Con relación a las violaciones de los derechos humanos el $13 \%$ manifiesta que si han sido objeto de violación a sus derechos, pero que nunca han denunciado porque no encuentran eco en las instituciones encargadas de velar por ellos y que el recurso económico también incide en no darle seguimiento por los demasiados gastos en pasajes para viajar a Jutiapa.

Y en relación que si conocen las instituciones que velan por sus derechos humanos del $100 \%$ encuestado el $38 \%$ manifiestan desconocer cuales son las instituciones que les prestan ayuda en el momento de sentirse con problemas en materia de derechos humanos, y que tienen el problema que únicamente existen en Jutiapa como Procuraduría de Derechos Humanos o la Procuraduría General de Nación, y desconocían que también podían hacerlo en la Policía Nacional Civil o en la Oficina de la Mujer en las municipalidades.

\section{Referencias}

Aguirre L. (1987), Si quieres la paz, ed. Mosca hermanos. Montevideo pag. 16.

Campos G. (1989), teoría general de los derechos humanos v edición 120 México.

De las mujeres ed. I Buenos Aires, Argentina.

Espinoza P. (2005:17), conferencia inaugural de la cumbre global de los derechos

Garcia E. (1994), la teoría de Justicia y Derechos Humanos.

Garcia E. (1999), Derechos Humanos y calidad de vida, en Graciano González 4.

Guerrero. P (1999:17), El estado como sujeto de los derechos económicos, políticos.

Gonzalez. (1999):12, Derechos Humanos, la condición humana en la sociedad.

Luno A. (2006), la tercera generación de los derechos humanos.

Luño A. (2005), Estado de derecho y Constitución editorial 5 México distrito federal.

Mirándola.(2009):30, discurso sobre la dignidad del hombre, México, UNAM,2009.

Pp30, monitoreo de medios, Asociación mundial para las comunicación-Ciones.

Nino C. (1989), Ética y Derechos Humanos, Barcelona Ariel.

Pinto M. (1997), temas de derechos humanos I edición, buenos aires argentina.

Reyes J. (1999):11, la escuela sola no hara de las escuelas el papel de la educación No 
formal, educación ambiental octubre de 1999.

Rodríguez, (2006):11, laeducaciónyPedagogía.

Roosevet E. (1962), los derechos humanos atravez de la historia.

Salcedo J. (2001), soberanía de los estados Derechos Humanos.

Véase Bustan la sociedad tecnológica Madrid Tecnos. Y humanos, nueva época, año 6 numero 17.

\section{Sobre autora}

\section{Randolfo Alfaro Navas}

Profesor Titular I de la Facultad de Humanidades Sede 88 Yupiltepeque, Licenciado en Pedagogía en Administración educativa, Facultad de Humanidades, Sede Jutiapa, cierre pensum en Derechos Humanos sede Jalapa, Maestro en docencia Universitaria conÉnfasis enAndragogia, egresado del Centro Universitario del suroriente, Consurori Jalapa, actualmente imparto los cursos de Técnicas de Investigación Bibliográficas, 1er. Ingreso PEM en económico contable, Administración Pública y Privada, 3er. Ingreso PEM en pedagogía en Administración Educativa, Planificación Curricular en 2do. Ingreso PEM en Pedagogía en Administración Educativa, Elaboración de Proyectos de Investigación 3er. Ingreso PEM en Investigación Educativa y Diseño e Innovación Curricular en 2da. Cohorte Licenciatura en Pedagogía en Administración Educativa, acabo de entregar el cargo de prosecretario en el Colegio Profesional de Humanidades, y he obtenido diplomados en Derechos Humanos, filosofía y en económico contable.

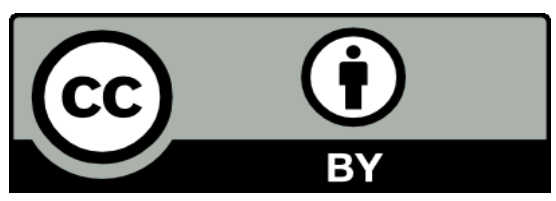

Este texto está protegido por una licencia CreativeCommons 4.0.

Esta licencia permite que otros distribuyan, mezclen, adapten y desarrollen su trabajo, incluso comercialmente, siempre y cuando le den crédito por la creación original. 